\title{
Synthesis of composite thin-film polymer consisting of tungsten and zinc oxide as hydrogen gas detector
}

\author{
Alister G. Willis ${ }^{1, *}$, and Saharudin Haron ${ }^{1}$ \\ ${ }^{1}$ Centre of Hydrogen Economy, Institute of Future Energy, Universiti Teknologi Malaysia, 81310 \\ Johor Bahru, Johor, Malaysia.
}

\begin{abstract}
A composite polymer consisting of polyaniline (PANI) was synthesised via oxidative polymerisation by varying concentrations of transitional metal oxides and the presence of a hydrogen dissociation catalyst, palladium (Pd). The metal oxides chosen for this study were tungsten oxide $\left(\mathrm{WO}_{3}\right)$ and zinc oxide $(\mathrm{ZnO})$. The composite polymer samples were characterised using Fourier transform infrared (FTIR) spectroscopy where ultraviolet-visible (UV-Vis) spectroscopy was used to observe the optical changes of the thin films due to exposure to hydrogen. The FTIR spectra obtained confirmed the synthesis of PANI composite. Based on the UV-VIS analysis, PANI-ZnO composite polymer showed the highest difference in peak intensity before and after exposure to hydrogen with $11.4 \%$ difference.
\end{abstract}

\section{Introduction}

Hydrogen $\left(\mathrm{H}_{2}\right)$ has gained attention in clean energy research as a fuel source even with the safety risk involved [1]. Firstly, $\mathrm{H}_{2}$ has a wide range of flammability and capable of leaking through pipelines or containing vessels due to small molecular size [2]. Secondly, it is difficult to detect $\mathrm{H}_{2}$ because it is colourless and odourless. Most industrial processes such as energy production require $99 \%$ and above purity of $\mathrm{H}_{2}$, where adding additives into the gas as a leak indicator is not applicable for such system. The growth of $\mathrm{H}_{2}$ economy will lead to the development of massive infrastructures composed of large-scale storage tanks, liquid hydrogen tankers, electrolysis plants, long pipelines, and fuel cell power. Therefore, detection and quantitative estimation of $\mathrm{H}_{2}$ in a reliable and efficient manner is of excellent value in these applications, not only from a safety standpoint but also economically beneficial [3].

Detection technologies are based on the measurable changes of the sensing material properties. These changes can be physical or chemical such as resistance, electrical current, temperature, dielectric properties, transmittance, bending, and frequency. Currently,

* Corresponding author: agenndi2@live.utm.my 
hydrogen sensors can be classified into several categories based on the detection mechanism such as catalytic, thermal conductivity, resistivity, electrochemical, acoustic, and optical.

Catalytic sensors are based on the heat output due to the reaction between $\mathrm{H}_{2}$ and oxygen $\left(\mathrm{O}_{2}\right)$ on the surface of the sensor. This type of sensor requires heating of the catalytic bead, which means the sensors are power dependent. Thermal conductivity works on the principle of heat loss from a hot region to the surrounding gas. Due to this principle, this type of sensor can be influenced by ambient temperature fluctuation. A resistive $\mathrm{H}_{2}$ sensor is based on the resistance change of palladium (Pd). Increased electrical resistance arises due to the formation of palladium hydride. An electrochemical hydrogen sensor is based on the electrochemical reaction at the electrode. Low concentration of $\mathrm{H}_{2}$ can be detected using this type of sensor and does not require heating [4]. Acoustic sensors detect the acoustic property differences when exposed to $\mathrm{H}_{2}$. For optical sensors, the sensing materials change its optical properties when react with $\mathrm{H}_{2}$. Commonly used materials are palladium, which reacts with $\mathrm{H}_{2}$ and changes its optical properties due to structural differences. Over the years, research has been done to improve the existing sensing materials such as faster response time, higher sensitivity, and longer lifetime. Since the discovery of conducting polymer in the mid- $19^{\text {th }}$ century [5], these polymers have shown the capability to enhance sensing materials and the possibility to substitute conventional sensing materials.

Polyaniline (PANI) is an intrinsically conducting polymer that consists of two repeating units formed by amine or imine [6]. Conventional synthesis of PANI involves oxidative polymerisation of aniline, which leads to emeraldine salt form [7]. PANI can exist in three different oxidation states [8], namely leucoemeraldine (fully reduced), emeraldine (half oxidised), and pernigraniline (fully oxidised) [9]. In emeraldine form, PANI can drastically change its properties through protonation or deprotonation [10]. Emeraldine base, which is the unprotonated form, is an electrical insulator but with protonation, it is converted to emeraldine salt that conducts electricity close to metals. In this study, composite PANI consisted of transitional metal oxide and Pd was investigated for its optical properties after exposure to $\mathrm{H}_{2}$. The addition of $\mathrm{Pd}$ is to assist in splitting $\mathrm{H}_{2}$ to $\mathrm{H}^{+}$, which is a proton. This study attempted to utilise hydrogen atom as a source for emeraldine base protonation.

\section{Experimental methods}

\subsection{Materials}

All materials used in this study were obtained from Sigma-Aldrich Co. The materials were tungsten oxide $\left(\mathrm{WO}_{3}\right)(99.9 \%)$, zinc oxide $(\mathrm{ZnO})(99.9 \%)$, aniline $(99 \%)$, and methanol $(99.8 \%)$, as well as palladium chloride $\left(\mathrm{PdCl}_{2}\right)$, sodium hydroxide $(\mathrm{NaOH})$, hydrochloric acid ( $\mathrm{HCl}$ ), ammonium persulfate (APS), acetone, N-methyl-2-pyrrolidone (NMP), and distilled water.

\subsection{Preparation of PANI-ZnO}

$\mathrm{ZnO}(0.2,0.4,0.9$, and 1.6 g equivalent of 5, 10, 20, and $30 \mathrm{wt} . \%)$ was dissolved in $400 \mathrm{ml}$ of $0.1 \mathrm{M}$ aniline in $1 \mathrm{M} \mathrm{HCl}$ and stirred for $30 \mathrm{~min} .1 \% \mathrm{w} / \mathrm{v}$ of $\mathrm{PdCl}_{2}$ to aniline was added dropwise into the solution of $0.05 \mathrm{M}$ APS in $1 \mathrm{M} \mathrm{HCl}$ while stirring over the period of 30 
min. Moderate stirring was continued for $8 \mathrm{~h}$ and then the mixture was left to settle overnight. The precipitates were filtered and washed with distilled water until they reached the $\mathrm{pH}$ of filtrate. The precipitates were also washed with methanol and acetone to remove oligomers and unreacted monomers. Meanwhile, the polymer was washed with distilled water and left to dry in air for over $24 \mathrm{~h}$. The obtained powder was subjected to $1 \mathrm{M} \mathrm{NaOH}$ treatment for conversion to emeraldine base form. The final product of PANI was dissolved in NMP to form $1 \mathrm{wt}$. \% casting solution. The solution was drop casted on a glass substrate and the solvent was left to evaporate completely from the substrate.

\subsection{Preparation of $\mathrm{PANI}-\mathrm{WO}_{3}$}

PANI was prepared through oxidative polymerisation using a standard procedure [10] with the presence of $\mathrm{WO}_{3}$ and $\mathrm{Pd} .0 .1 \mathrm{M}$ aniline was prepared in $1 \mathrm{M} \mathrm{HCl}$. $\mathrm{WO}_{3}$ was added to the solution in different weight percentages. $1 \% \mathrm{w} / \mathrm{v}$ of $\mathrm{PdCl}_{2}$ to aniline was added into the solution. The aniline solution was subjected to sonication for $20 \mathrm{~min}$. The solution then was stirred using a magnetic stirrer whereas 0.05 M APS was added dropwise over the period of $6 \mathrm{~h}$. The precipitate was left to settle overnight. The precipitate was filtered and washed with acetone and deionised water until the filtrate became clear. The obtained solid was dried in an oven and ground to powder. $\mathrm{WO}_{3}$-PANI was treated with $1 \mathrm{M} \mathrm{NaOH}$ to convert PANI in salt form into base form. The final product of PANI was dissolved in NMP to form 1 wt. \% casting solution. The solution was drop casted on a glass substrate and the solvent was left to evaporate completely from the substrate.

\subsection{Characterisation}

The obtained powder was characterised using Fourier transform infrared (FTIR) spectroscopy to confirm the synthesis of PANI. The optical properties of PANI composite were investigated using ultraviolet-visible (UV-Vis) spectroscopy. Eight different compositions were tested for this study, which were labelled as W5, W10, W20, and W30 for PANI-WO $\mathrm{W}_{3}$ and Z5, Z10, Z20, and Z30 for PANI-ZnO, which correspond to the weight percentages. UV-Vis spectra were obtained before and after exposure to $\mathrm{H}_{2}$ in order to observe the response. $\mathrm{H}_{2}$ was set at $0.5 \mathrm{~L} \mathrm{~min}^{-1}$ and passed through the test chamber.

\section{Results and discussion}

\subsection{FTIR spectra of PANI-WO}

FTIR spectra of PANI- $\mathrm{WO}_{3}$ composite shown in Figure 1 were observed for each sample in the range of $500-3600 \mathrm{~cm}^{-1}$ to confirm the polymerisation of PANI. All samples showed high peaks in the region of $750-1600 \mathrm{~cm}^{-1}$, which are the characteristics of PANI [11]. The main $\mathrm{WO}_{3}$ vibrations were found in the regions of $600-1453 \mathrm{~cm}^{-1}$ and $3200-3400 \mathrm{~cm}^{-1}$, which correspond to its bending, stretching, and lattice modes [12]. 


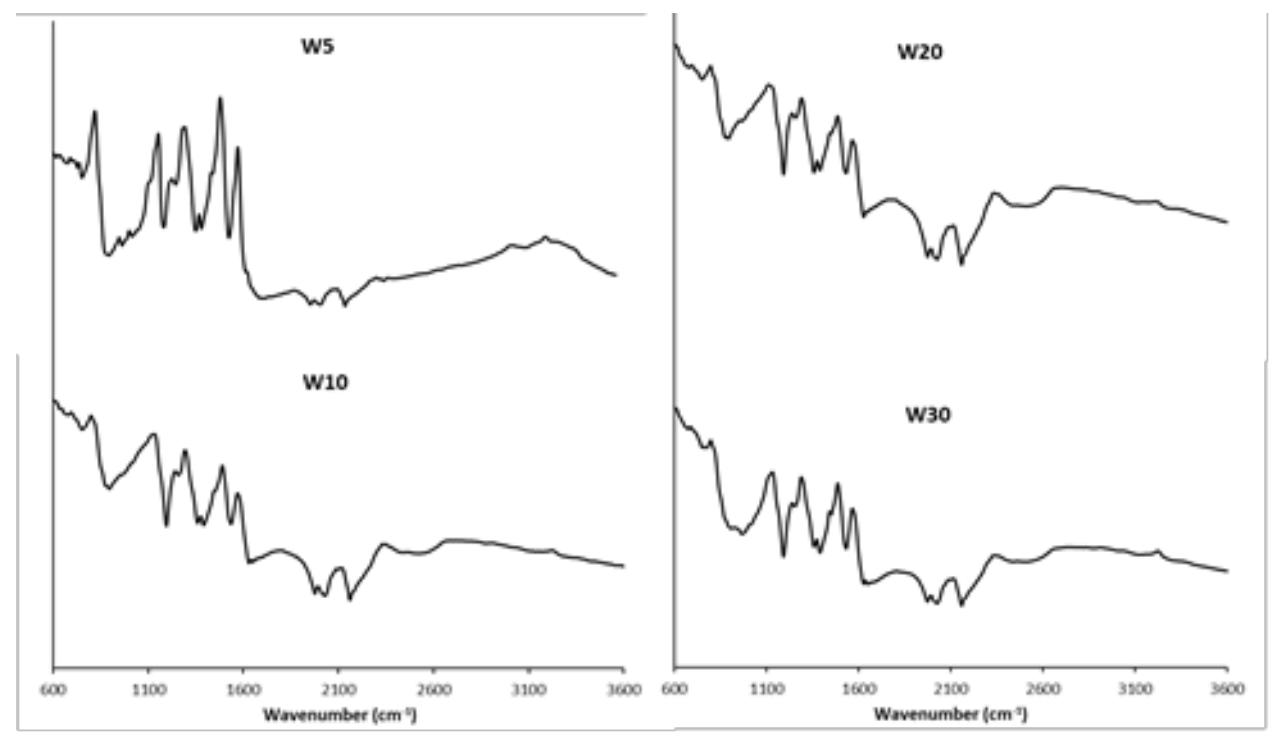

Fig. 1. FTIR spectra of PANI-WO $\mathrm{W}_{3}$

\subsection{FTIR spectra of PANI-ZnO}

FTIR spectra of PANI-ZnO composite were observed for each sample in the range of 600 $3600 \mathrm{~cm}^{-1}$ to confirm the polymerisation of PANI as shown in Figure 2. The spectra exhibited characteristic bands at 3200-3300, 2113, 1637, 1583, 1491, 1289, 1221, 1162, 1109-893, 826, and $450 \mathrm{~cm}^{-1}$ [13]. The band at $3200-3300 \mathrm{~cm}^{-1}$ is due to the amine stretching of benzenoid. The peaks at 1583 and $1491 \mathrm{~cm}^{-1}$ are attributed to the $\mathrm{C}=\mathrm{C}$ stretching of quinoid and benzenoid rings, respectively. The emeraldine base structure is indicated by the peaks at $1289 \mathrm{~cm}^{-1}$. Furthermore, the band at $1162 \mathrm{~cm}^{-1}$ is due to the imine unit in the quinoid ring of PANI-ZnO nanocomposite [14].

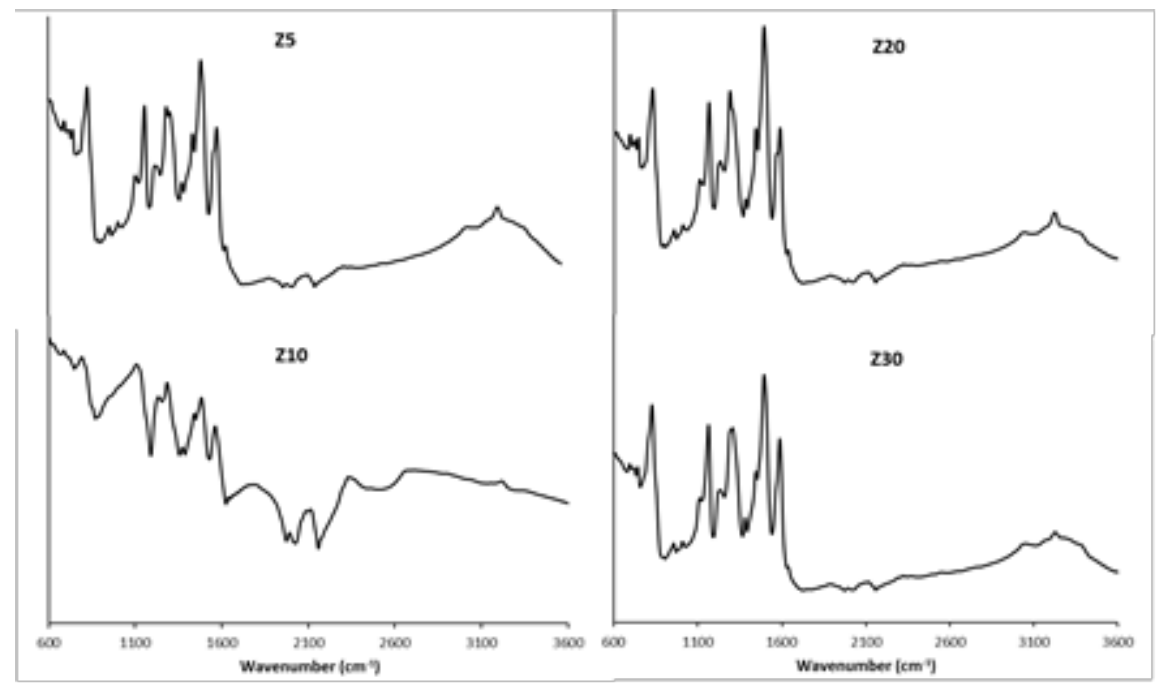

Fig. 2. FTIR spectra of PANI-ZnO. 
The samples were analysed using a UV-Vis spectrophotometer to measure the peak before and after the samples were exposed to $\mathrm{H}_{2}$. The results of UV-Vis test were evaluated between the ranges of 190 and $1100 \mathrm{~nm}$. Then, the range was scaled down at the peak of $600 \mathrm{~nm}$, which corresponds to the exciton formation in the imine nitrogen [15]. Figure 3 shows the result of $\mathrm{PANI}-\mathrm{WO}_{3}$ composite samples before exposure to $\mathrm{H}_{2}$. It was observed that the increase of $\mathrm{WO}_{3}$ caused the peak intensity to increase as well.

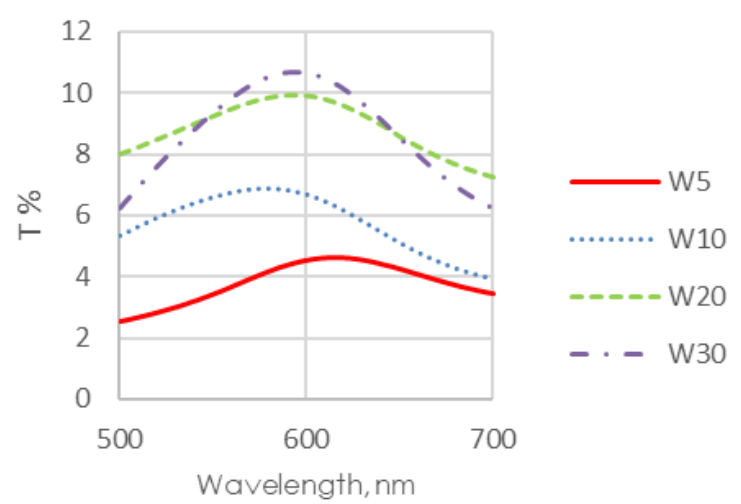

Fig. 3. UV-Vis spectra of $\mathrm{PANI}-\mathrm{WO}_{3}$ before $\mathrm{H}_{2}$ exposure.

Figure 4 shows the result of UV-Vis spectroscopy for $\mathrm{PANI}_{-}-\mathrm{WO}_{3}$ after it passed through $\mathrm{H}_{2}$. The results showed that the peak intensity of the samples increased after exposure to $\mathrm{H}_{2}$. The increased intensity indicates the relative amount of quinoid imine unit in PANI molecules [16]. After exposure to $\mathrm{H}_{2}, \mathrm{H}^{+}$delocalised the amine unit, which leads to the increased relative amount of quinoid imine unit [17]. This increment explains the increased peak intensity.

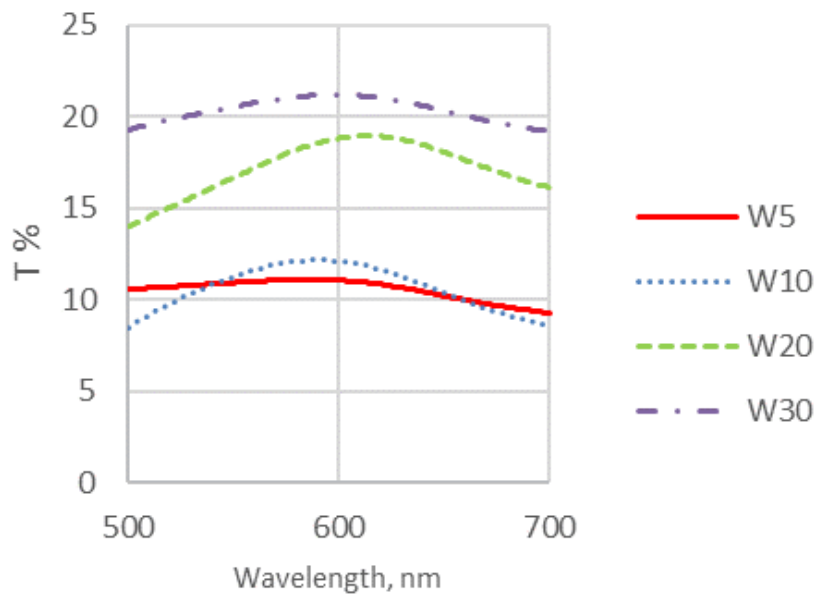

Fig. 4. UV-Vis spectra of $\mathrm{PANI}-\mathrm{WO}_{3}$ after $\mathrm{H}_{2}$ exposure.

Figure 5 shows the difference of peak intensity for $\mathrm{PANI}_{-}-\mathrm{WO}_{3}$ before and after the samples passed through $\mathrm{H}_{2}$. W30 had the highest peak difference of up to $11.3 \%$ compared to others. It can be concluded that W30 is the best composition to be used as a hydrogen sensor as it has the highest detection for $\mathrm{PANI}_{-} \mathrm{WO}_{3}$ composite compared to others. 


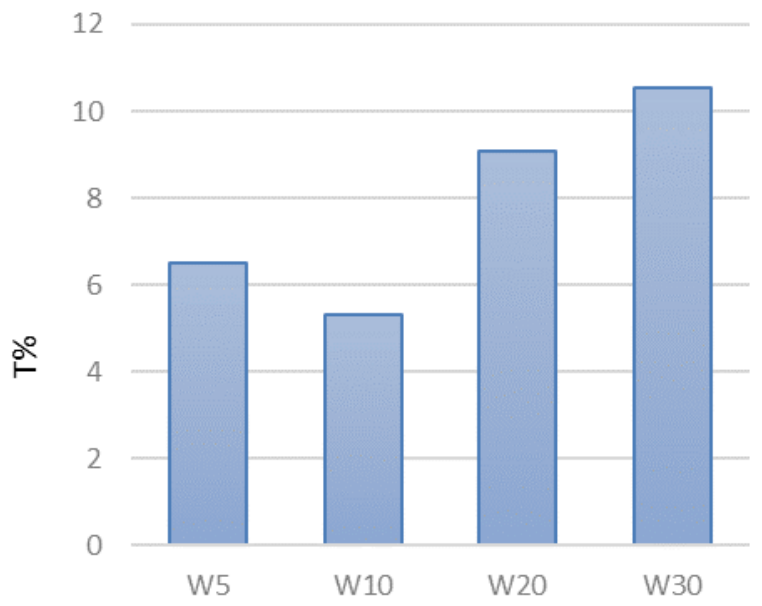

Fig. 5. Difference in peak intensity for PANI- $\mathrm{WO}_{3}$.

Figure 6 shows the overall result of PANI-ZnO samples before exposure to $\mathrm{H}_{2}$. Based on the results, an increase of $\mathrm{ZnO}$ content in the composite reduced the transmittance. After that, the samples were placed inside a vessel. Figure 7 shows the UV-Vis spectra of the samples after exposure to $\mathrm{H}_{2}$. It was observed that all samples showed an increased peak intensity whereas Z30 peak seemed to be shifted to the left. A further study is needed to investigate the shifting of this peak.

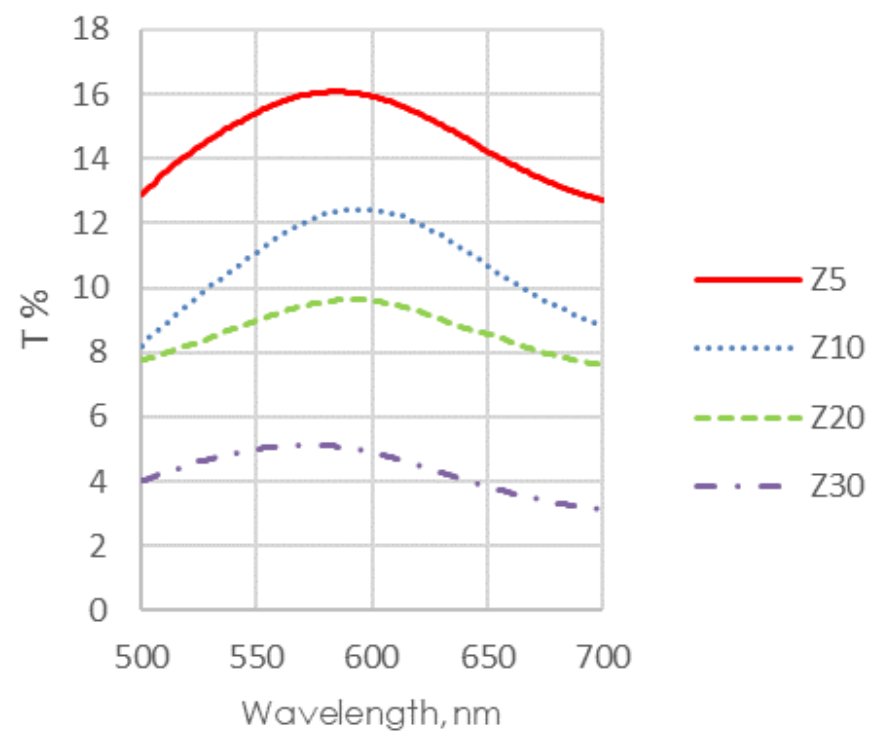

Fig. 6. UV-Vis spectra of PANI-ZnO before $\mathrm{H}_{2}$ exposure. 


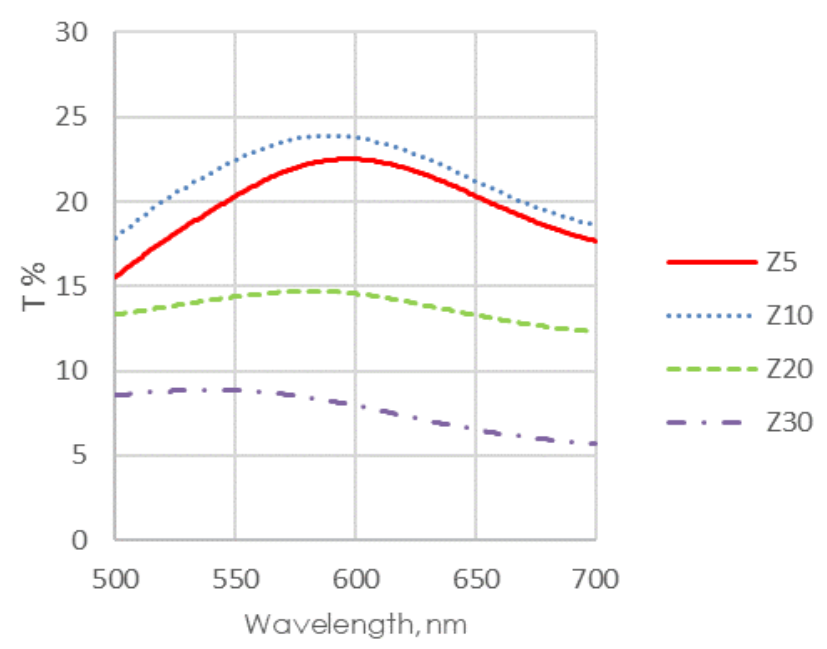

Fig. 7. UV-Vis spectra of PANI-ZnO after $\mathrm{H}_{2}$ exposure.

Figure 8 shows the peak difference for PANI-ZnO before and after the samples were exposed to $\mathrm{H}_{2}$. $\mathrm{Z} 10$ had the highest difference up to $11.4 \%$ compared to others. It can be concluded that Z10 is the best composition for a hydrogen sensor as it has the highest detection for PANI-ZnO composite compared to others. The findings are similar to a study where decreased peak intensity occurred when the weight percentage of $\mathrm{ZnO}$ increased. This indicates the reduction of singlet exciton generation due to the presence of $\mathrm{ZnO}$ and might interact with the interchain of PANI species [18].

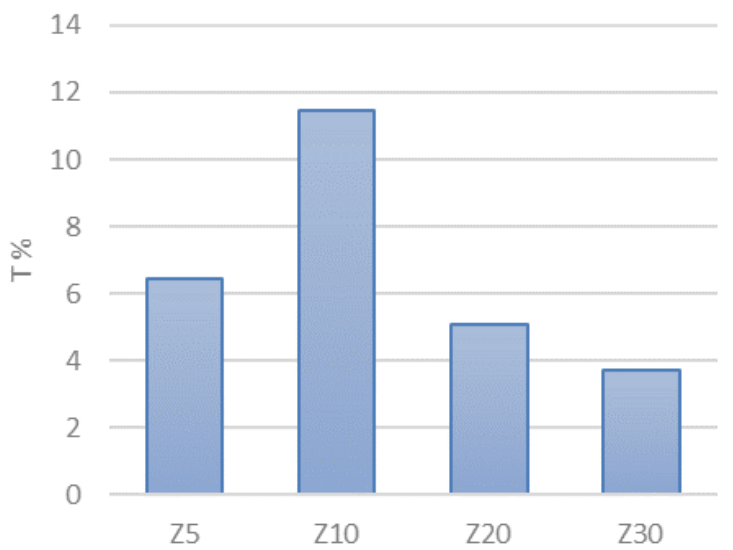

Fig. 8. Difference in peak intensity for PANI-ZnO.

Based on the results obtained, a comparison was made between PANI-ZnO and PANI-WO samples. The samples showed a distinct difference between the two types of composite, where increased content of $\mathrm{WO}_{3}$ increased the peak intensity while it was the opposite for PANI-ZnO samples. However, both composites showed an increase in peak intensity after exposure to $\mathrm{H}_{2}$, with sample $\mathrm{Z} 10$ showed the highest response among all the samples. Therefore, a further study is needed to optimise the performance of the sensing film.

\section{Conclusion}




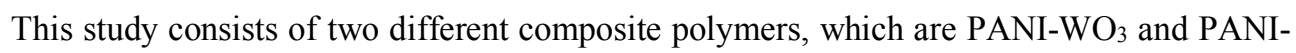
$\mathrm{ZnO}$ composite polymers. The samples were successfully prepared and characterised using FTIR spectroscopy and UV-Vis spectroscopy. For FTIR analysis, the peaks obtained were discussed and it is shown that the spectra are similar with a previous study. The characterisation of the composite polymers by FTIR spectra identified that different weight percentages of metal oxide resulted in similar absorption peaks. Based on UV-Vis analysis, it was shown that increased of $\mathrm{ZnO}$ content in the composite caused the decrease of peaks. In contrast, an opposite pattern was detected for PANI-WO $\mathrm{W}_{3}$ composite. Based on the results, Z10 showed the highest difference in peak intensity before and after exposure to $\mathrm{H}_{2}$ with $11.4 \%$ difference.

This work was financially supported by Universiti Teknologi Malaysia under the Research University Grant Q.J130000.2646.15J62 and the Ministry of Higher Education Malaysia.

\section{References}

1. R. Chen, X. Ruan, W. Liu, C. Stefanini, Int. J. of Hydrog. Energy, 40(1), 746-751 (2015)

2. A. Kumar, P. Zhang, A. Vincent, R McCormack, R. Kalyanaraman, H. J. Cho, S. Seal, Sens Actuators B Chem., 155(2), 884-892 (2011)

3. T. Hubert, L. Boon-Brett, G. Black, U. Banach, Sens Actuators B Chem., 157(2), 329$352(2011)$

4. W. Li, D.M. Jang, S.Y. An, D. Kim, S. Hong, H. Kim, Sens Actuators B Chem., 160(1), 1020-1025 (2011)

5. G. Inzelt, Conducting Polymers ( Springer, Berlin, Heidelberg, 2008)

6. W.S. Huang, B.D. Humphrey, A.G. MacDiarmid, J. Chem. Soc., 82, 2385 (1986)

7. A.G. MacDiarmid, J.C. Chiang, A.F. Richter, A.J. Epstein, Synth. Met., 18, 285 (1987)

8. J. Stejskal, P. Kratochvil, N. Radhakrishnan, Synth. Met., 61, 225-231 (1993)

9. A. Ray, G.E. Asturias, D.L. Kershner, A.F. Richter, A.G. MacDiarmid, Synth. Met., 29, $141(1989)$

10. Y. Cao, P. Smith, A.J. Heeger, Synth. Met., 32, 263 (1989)

11. J. Diaz-Reyes, V. Dorantes-Garcia, A. Perez-Benitez, J.A. Balderas-Lopez, Superf. vacio, 21(2), 12-17 (2008)

12. P. R. Somani, R. Marimuthu, A.B Mandale, Polymer, 42(7), 2991-3001 (2001)

13. T. Mathavan, J. Archana, Y. Hayakawa, K. Anitha, M.A. Jothirajan, A. Divya, A. Milton, Int. J. ChemTech Res., 7(3), 1253-1258 (2014)

14. F. Alvi, M.K. Ram, H. Gomez, R.K. Joshi, A. Kumar, Polym. J., 42(12), 935 (2010)

15. A.P. Monkman, J.L. Bredas, R.R. Chance, Conjugated polymeric materials (Kluwer, Dordrecht, 1990)

16. J.F. Wolf, C.E. Forbes, S. Gould, L.W. Shacklette, J. Elect. Soc., 136(10), 2887 (1989)

17. J.E. Albuquerque, L.H.C Mattoso, D.T. Balogh, R.M. Faria, J.G. Masters, A.G. MacDiarmid, Synth. Met., 113, 19 (2000)

18. S. Ameen, M.S. Akhtar, Y.S. Kim, O. Yang, H. Shin, Colloid Polym. Sci., 289(14), 415-421 (2011)

\section{Acknowledgement}


This work was financially supported by the Universiti Teknologi Malaysia under the Research University Grants Q.J130000.2646.15J62 and Ministry of Higher Education Malaysia. 